\title{
ON THE INITIAL EXPANSION OF METEOR TRAILS
}

\author{
V.C. LIU and S.J. YING \\ The University of of Michigan, Ann Arbor, Michigan, USA
}

Received 24 December 1975

\begin{abstract}
The asymptotic solution of the equation governing the number density $n(r, t)$ of evaporated meteoric atons behind a steadily moving meteor is obtained which is suitable to depict the initial expansion of a meteor trail. Some unusual results of meteor observations are elucidated.
\end{abstract}

When a rapidly moving meteorite enters into the earth's atmosphere intensive aerodynamic heating is developed such that meteor atoms evaporate from the heated surface. The atomic collisions in a meteor trail of the particles, either evaporated or reflected, from the meteorite with the ambient atmosphere cause dissociation, excitation and ionization of the participating atomic species. The presence of free electrons and luminosity thus produced in the meteor trail are basic to the Radar-Echo and spectroscopic studies of meteor respectively. The importance of meteorites as the upper atmospheric probes is well known in geophysical research. Meteorites are also studied in order to learn about the physical and chemical history of at least a small class of extraterrestrial solar system objects. As the evaporated meteortic atom traveling, at say, $50 \mathrm{~km} / \mathrm{sec}$ encounter atmospheric atoms which have thermal velocities of the order of $\frac{1}{2} \mathrm{~km} / \mathrm{sec}$, they will retain on the average a fraction of their initial speeds with, however, changes of directions. After several collisions the meteoric atoms will have acquired sufficiently random directions of travel so that the theory of random walks (or diffusion) may be applied to their ensuing paths. The kinetic behavior of the meteoric atoms in their pre-diffusion stage has important bearings on the detectability of meteors via Radar-Echo [1] and the atomic collision processes in the meteor trails [2] . A recent contribution to the kinetics of meteor trails gives a new equation which governs the evolution of the density $n(r, t)$ of the meteoric atoms in the form of the telegraph equation $[3,4]$ :

$\nabla^{2} n=A^{-2} n_{t t}+D^{-1} n_{t}$ where $A^{2}=k T / m$ and $D=k T / m \nu$ with $T$, assumed constant, denoting the effective kinetic temperature of the evaporated meteoric atoms of mass $m$ in collision with the ambient atmosphere at frequency $\nu ; k$, the Boltzmann constant. Notice that eq. (1) depicts a meteor trail which spreads in the nature of wave propagation and diffusion as its short- and long-time behavior respectively. The application of eq. (1) has been limited to an idealized one-dimensional stationary model [3-6]; hence is not suited for the quantitative interpretation of meteor observations. A more realistic three-dimensional model is constructed herein.

Consider a meteor of radius $r_{0}$ moving at a constant velocity $-V$ along the $z^{\prime}$-axis of a cylindrical coordinate $\left(r, \theta, z^{\prime}\right)$. Assume that meteoric atoms are emitted uniformly at the surface $\left(r=r_{0}\right)$ of the moving meteor with a constant rate. In a moving coordinate where $z=z^{\prime}+V t$, eq. (1) becomes

$n_{r}+r^{-1} n_{r}=\left(M^{2}-1\right) n_{z z}+(V / D) n_{z}$,

where $M=V / A$, with the following boundary conditions:

$n_{z}=n=0$ at $z=0 ; \quad n=0$ as $r \rightarrow \infty$;

$n_{r}=-n_{0}^{\prime} \quad$ at $r=r_{0}$ for all $z$.

The use of Laplace transformations such that $N(r, s)=\int_{0}^{\infty} \mathrm{e}^{-s z} n(r, z) \mathrm{d} z$, to eqs. (2) and (3) results, after simplifications,

$N=\frac{n_{0}}{s \beta} \frac{K_{0}(\beta r)}{K_{1}(\beta r)}$

where $\beta=\left[\left(M^{2}-1\right) s^{2}+V s / D\right]^{1 / 2} ; K_{0}$ and $K_{1}$, the 
modified Bessel functions of the second kind. In as much as our primary interest herein is in the initial expansion of the meteor trail, hence the solution of the above-initial value problem at the small values of $z$, we first obtain the asymptotic expansion of $K_{0}(\beta r)$ and $K_{1}(\beta r)$ in eq. (4) for large $s$ and keep terms of the order $\beta^{-1 / 2} \mathrm{e}^{-\beta r}$ only. Thus

$$
\begin{aligned}
N= & n_{0}^{\prime}\left(M^{2}-1\right)^{-1 / 2}\left(r_{0} / r\right)^{1 / 2} s^{-1} F^{-1} \\
& \times \exp \left[-\left(r-r_{0}\right)\left(M^{2}-1\right)^{1 / 2} F\right] .
\end{aligned}
$$

where $F=\left\{\left[s+V / 2 D\left(M^{2}-1\right)\right]^{2}-\left[V / 2 D\left(M^{2}-1\right)\right]^{2}\right\}^{1 / 2}$ and then transform $N(r, s)$ inversely into

$$
\begin{aligned}
n= & \frac{n_{\theta}^{\prime}}{\left(M^{2}-1\right)^{1 / 2}}\left(\frac{r_{0}}{r}\right)^{1 / 2} \\
& \times \int_{0}^{z} \exp (-\alpha z) I_{0}\left[\alpha\left(z^{2}-R^{2}\right)^{1 / 2}\right] H(z-R) \mathrm{d} z,
\end{aligned}
$$

where $\alpha=V / 2 D\left(M^{2}-1\right) ; R=\left(r-r_{0}\right)\left(M^{2}-1\right)^{1 / 2} ; I_{0}$, modified Bessel function of the first kind; $H$, Heaviside unit function, which, for small $z$ with $I_{0}=1$, can be reduced to

$$
\begin{aligned}
n= & \frac{2 n_{0}^{\prime} D}{V}\left(M^{2}-1\right)^{1 / 2}\left(\frac{r_{0}}{r}\right)^{1 / 2} \\
& \times\left\{\exp \left[-\alpha\left(r-r_{0}\right)\right]-\exp [-\alpha z]\right\} H(z-R) .
\end{aligned}
$$

Notice that solution (7) depicts the field with a conical shaped wave front having its vertex angle depending on the value $\left(M^{2}-1\right)$ much like that of the flow field behind a Mach cone of a supersonically moving slender body. It we can assume that the distribution of the charged particles, produced from the meteoric atomic collisions, in the trail is closely related to that of the meteoric atoms, Halliday's observation of the initial "explosively" fast expanding trail of the Geminid meteor [5-7] can be elucidated in terms of the wave-propagation characteristics of eq. (7). The observed concentric double trails behind fast meteors [5-8] can be interpreted from the present theory by identifying the outer trail as caused by the much higher energetic collisions between the ambient atmosphere and the atmospheric atoms specularly reflected from the meteor surface. Notice that the value of $A$ corresponding to these reflected molecules are much higher than that of the evaporated meteoric atoms, hence their trail has a larger conical angle, thus a wider tail. Whether the outer trail is atmospheric in nature can be determined from the results of spectroscopic analyses of the trail luminosity.

It is of interest to note the mathematical similarity between the present kinetic theory of meteor trails and that of neutron thermalization $[9,10]$.

This work is supported by the National Aeronautics and Space Administration under Grant NGR 23-005094.

\section{References}

[1] J.S. Greenhow and J.E. Hall, M.N Roy, Astron. Soc. 121 (1960) 183.

[2] H.S.W. Massey and D.W. Sida, Phil. Mag. 46 (1955) 190.

[3] V.C. Liu, Phys. Fluids 13 (1970) 62.

[4] V.C. Liu, Space Sci. Rev. 9 (1969) 423.

[5] V.C. Liu, Proc. 7th Int. Symp. on Rarefied Gas Dynamics, Pisa (1970).

[6] V.C. Liu, Prog. Aerospace Sci. 16 (1975) 273.

[7] I. Halliday, Smithson. Contr. Astrophys. 7 (1963) 171.

[8] B.A. Mirtov, Soviet Astron. A.J. 4 (1960) 455.

[9] M.M.R. Williams, The slowing down and thermalization of neutrons (North-Holland, Amsterdam, 1966)

[10] I. Kuscer, Advances in neutron thermalization theory, Symp. on Neutron Thermalization and Reactor Spectra. I.A.E.A. 1 (1967) 3. 